\title{
Face Recognition Technique Using Symbolic PCA Method
}

\author{
P.S. Hiremath ${ }^{1}$ and C.J. Prabhakar ${ }^{2}$ \\ ${ }^{1}$ Department of Studies in Computer Science, Gulbarga University, \\ Gulbarga - 585106, Karnataka, India \\ hiremathps@yahoo.co.in \\ 2 Department of Studies in Computer Science, Kuvempu University, \\ Shankaraghatta - 577451, Karnataka, India \\ psajjan@yahoo.com
}

\begin{abstract}
A face recognition technique based on symbolic PCA approach is presented in this paper. The proposed method transforms the face images by extracting knowledge from training samples into symbolic objects, termed as symbolic faces. Symbolic PCA is employed to compute a set of subspace basis vectors for symbolic faces and then to project the symbolic faces into the compressed subspace. New test images are then matched with the images in the database by projecting them onto the basis vectors and finding the nearest symbolic face in the subspace. The feasibility of the new symbolic PCA method has been successfully tested for face recognition using ORL database. As compared to eigenface method, the proposed method requires less number of features to achieve the same recognition rate.
\end{abstract}

\section{Introduction}

Face recognition involves computer recognition of personal identity based on statistical or geometrical features derived from the face images. Face recognition technology is primarily used in law enforcement applications especially mug shot albums (static matching) and video surveillance (real time matching by video image sequences). Template based face recognition techniques using the Principal Component Analysis was first proposed by Kirby and Sirovich [5] to efficiently represent pictures of human faces. Turk and Pentland [8] presented the well known eigenface method for face recognition in 1991. Since then, PCA has been widely investigated and has become one of the most successful approaches in face recognition.

The classical PCA (eigenface) approach uses data, which is single quantitative value, representing every pixel of a face image as a feature. The proposed method is based on symbolic data, where each feature can be viewed as quantitative multivalued symbolic variable [1]. This is because same face is captured in different orientation, lighting, expression and background which leads to number of poses of same face with same set of features measured in all poses and in the same sequence. This results in coverage of complete information about features of a face represented by a symbolic object. Features characterizing symbolic object may be large in number, which leads to creation of a multi-dimensional feature space. Larger the dimensionality, more severe is the problem of storage and analysis. Hence a lot of importance has been attributed to the process of dimensionality or feature reduction of symbolic objects, which is 
achieved by subsetting or transformation methods [4] [7]. Gowda and Nagabhushan [6] proposed a dimensionality reduction method on interval data based on Taylor series. Ichino [3] proposed an extension of a PCA based on a generalized Minkowski metrics in order to deal with interval, set valued structure data. Choukria, Diday and Cazes [2] proposed different methods, namely, Vertices Method (V-PCA), Centers Method and Range Transformation Method. Face recognition system using symbolic data analysis involves high number of features; V-PCA requires high computational cost. So to overcome this difficulty we propose to use centers method.

The objective of the present paper is to perform symbolic data analysis of face images stored in a database by representing the face image by symbolic object of interval valued variables. The proposed method first transforms the face images by extracting knowledge from training samples into symbolic faces, each symbolic face summarizing the variation of feature values through the different images of the same subject. Symbolic PCA extracts features from symbolic faces, called as symbolic PCA features, and these are used for recognition purpose. The efficacy of the method is demonstrated by conducting experiments on face images of ORL database.

\section{Symbolic Faces}

Consider the face images $\Gamma_{1}, \Gamma_{2}, \ldots, \Gamma_{n}$, each of size $N \times M$, from a face image database. Let $\Omega=\left\{\Gamma_{1}, \ldots, \Gamma_{n}\right\}$ be the collection of $n$ face images of the database, which are first order objects. Each object $\Gamma_{l} \in \Omega, l=1, \ldots, n$, is described by a feature vector $\left(\tilde{Y}_{1}, \ldots, \tilde{Y}_{p}\right)$, of length $p=N M$, where each component $\tilde{Y}_{j}, j=1, \ldots, p$, is a single valued variable representing the intensity values of the face image $\Gamma_{l}$. An image set is a collection of face images of $m$ different subjects; each subject has same number of images but with different orientations, expressions and illuminations. There are $m$ number of second order objects (face classes) denoted by $c_{1}, \ldots, c_{m}$, each consisting of different individual images $\Gamma_{l} \in \Omega$. We denote the set $E=\left\{c_{1}, c_{2}, \ldots, c_{m}\right\}$ and $c_{i} \subseteq \Omega, i=1, \ldots, m$. The feature vector of each face class $c_{i} \in E$ is described by a vector of $p$ interval variables $Y_{1}, \ldots, Y_{p}$, and is of length $p=N M$. The interval variable $Y_{j}$ of face class $c_{i}$ is described by $Y_{j}\left(c_{i}\right)=\left[\underline{x}_{i j}, \bar{x}_{i j}\right]$, where $\underline{x}_{i j}$ and $\bar{x}_{i j}$ are minimum and maximum intensity values, respectively, among $j^{\text {th }}$ pixels of all the images of face class $c_{i}$. This interval incorporates information of the variability of $j^{\text {th }}$ feature inside the $i^{\text {th }}$ face class. We denote $X\left(c_{i}\right)=\left(Y_{1}\left(c_{i}\right), \ldots, Y_{p}\left(c_{i}\right)\right)$. The vector $X\left(c_{i}\right)$ of symbolic variables is recorded for each $c_{i} \in E$, and can be described by a symbolic data vector which is called as symbolic face : $X\left(c_{i}\right)=\left(\alpha_{i 1}, \ldots, \alpha_{i p}\right)$, where $\alpha_{i j}=Y_{j}\left(c_{i}\right), \quad j=1, \ldots, p$. We represent the $m$ symbolic faces by a $m \times p$ matrix:

$$
\underline{X}=\left(\begin{array}{ccc}
\alpha_{11} & \cdots & \alpha_{1 p} \\
\vdots & \ddots & \vdots \\
\alpha_{m 1} & \cdots & \alpha_{m p}
\end{array}\right)=\left(\alpha_{i j}\right)_{m \times p} .
$$




\section{Symbolic PCA}

The Symbolic PCA takes as input the matrix $\underline{X}$ containing $m$ symbolic faces pertaining to the given set $\Omega$ of images. We use centers method [2], which essentially applies the classical PCA method to the centers $x_{i j}^{c} \in \mathfrak{R}$ of the interval $\left[\underline{x}_{i j}, \overline{x_{i j}}\right]$, that is,

$$
x_{i j}^{c}=\frac{\bar{x}_{i j}+\underline{x}_{i j}}{2} .
$$

where $j=1, \ldots, p \quad$ and $\quad i=1, \ldots, m$

The $m \times p$ data matrix $\underline{X}^{C}$ containing the centers $x_{i j}^{c}$ of the intervals $\alpha_{i j}$ for $m$ symbolic faces is given by:

$$
\underline{X}^{c}=\left(\begin{array}{ccc}
x_{11}^{c} & \cdots & x_{1 p}^{c} \\
\vdots & \ddots & \vdots \\
x_{m 1}^{c} & \cdots & x_{m p}^{c}
\end{array}\right) .
$$

The mean vector $\Psi$ of $\underline{X}^{C}$ is defined by $\Psi=\left[\Psi_{j}\right]$, where $\Psi_{j}=\frac{1}{m} \sum_{i=1}^{m} x_{i j}^{c}$, $j=1, \ldots, p$. Each row vector of $\underline{X}^{C}$ differs from the mean vector $\Psi$ by the vector $\Phi_{i}=\left(x_{i 1}^{c}, x_{i 2}^{c}, \cdots, x_{i p}^{c}\right)-\Psi$. We define the matrix $\Phi$ as $\Phi=\left[\Phi_{1}^{\prime}, \ldots, \Phi_{m}^{\prime}\right]$. The covariance matrix $C$ is obtained as $C=\Phi^{\prime} \Phi$. Then, we calculate the eigenvalues $\lambda_{1} \geq \lambda_{2} \geq, \ldots, \lambda_{m} \geq 0$ and the corresponding orthonormalized eigenvectors $y_{1}, y_{2}, \ldots, y_{m} \in \mathfrak{R}^{m}$ of the covariance matrix $C$. The eigenvectors of symbolic PCA can be obtained as $V_{m}=\Phi Y_{m}$, where $Y_{m}=\left(y_{1}, \ldots, y_{m}\right)$ is the $m \times m$ matrix with columns $y_{1}, \ldots, y_{m}$ and $V_{m}$ is the $p \times m$ matrix with corresponding eigenvectors $v_{1}, v_{2}, \ldots, v_{m}$, as its columns. The subspace is extracted from the $p \times m$ dimensional space by selecting $S$ number of eigenvectors, which contain maximum variance and are denoted by $v_{1}, v_{2}, \ldots, v_{S}$, corresponding to eigenvalues $\lambda_{1} \geq \lambda_{2} \geq, \ldots, \lambda_{s}$. The weights $W_{i k}$ for $i^{t h}$ symbolic face, $i=1, \ldots, m$, are computed as

$$
W_{i k}=v_{k}^{T}\left(x_{i j}^{c}-\Psi\right) \text {. }
$$

where $k=1,2, \ldots, S$. The weights of $i^{\text {th }}$ symbolic face form the feature vector $\left(W_{i 1}, \ldots, W_{i S}\right)$ of the of $i^{\text {th }}$ symbolic face. The weights of test image $I_{\text {test }}$ are computed by projecting the test image into face subspace as:

$$
W_{\text {testk }}=v_{k}^{T}\left(I_{\text {test }}-\Psi\right) \text {. }
$$




\section{Experimental Results}

The proposed symbolic PCA method is experimented on the face images of the ORL database and compared with eigenface method. In the training phase, firstly construction of symbolic faces (as explained in section 2) for the given training samples is done and then symbolic PCA is applied to obtain the features (as explained in section 3). Further, a nearest neighbor classifier is employed for classification.

The ORL face database (developed at the Olivetti Research Laboratory, Cambridge, U.K.) is composed of 400 images with ten different images for each of the 40 distinct subjects. All the images were taken against a dark homogeneous background with the subjects in an upright, frontal position, with tolerance for some tilting and rotation of up to about $20^{\circ}$. There is some variation in scale of up to about $10 \%$. The spatial and gray level resolutions of the images are $92 \times 112$ and 256 , respectively. Some typical images of one subject of ORL database are given in Fig 1.

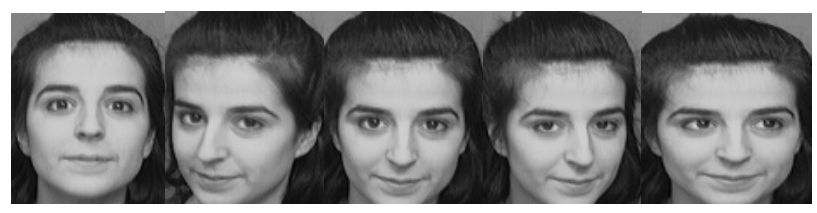

Fig. 1. Five sample images of one subject of the ORL Database

\subsection{Comparison of Variance in Eigenvectors of Symbolic PCA and Eigenfaces}

Firstly, the experiment is performed using all the 400 image samples (i.e., 40 face classes each with 10 face images) as training sample. Fig. 2 shows the comparison of percentage of variance in eigenvectors of symbolic PCA and eigenfaces. It is ob served that the first eigenvectors of symbolic PCA contain more information (energy) of training samples than the eigenfaces. Thus, fewer eigenvectors of symbolic PCA are required as compared to the large number of eigenfaces necessary to contain the same amount of information, which leads to the dimensionality reduction.

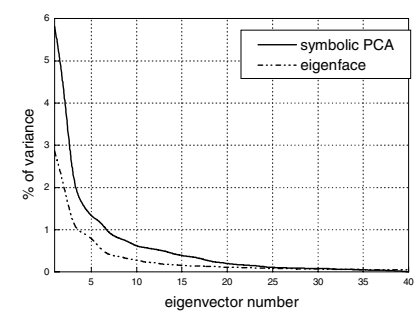

Fig. 2. Comparison of variance in eigenvectors of symbolic PCA and eigenfaces 


\subsection{Performance of Symbolic PCA Using Four Similarity Measures}

In recognition phase, a nearest neighbor classifier based on appropriate similarity measure is used. The experiments are performed using the first five image samples per face class for training and remaining images for testing (i.e., 200 training images and 200 testing images). The experiments are repeated for four different similarity measures, namely, City block (L1), Euclidean (L2), Mahalanobis and cosine similarity measures, and top recognition rates (\%) of 55.00, 49.25, 79.45, 92.15, respectively, are achieved. It is observed that the symbolic PCA performed with cosine similarity measure yields better recognition rate as compared to other similarity measures.

\subsection{Performance of Symbolic PCA Method for Varying Number of Features}

The experiments are performed by adopting leave-one-out strategy. The Fig. 3 shows symbolic PCA method with cosine similarity measure has recognition rate $85.5 \%$ using only 13 features, where as eigenface method with Euclidean similarity measure requires 42 features to achieve the same recognition rate. Further, the symbolic PCA with cosine measure achieves a recognition rate of $94.15 \%$ using 40 features. This is due to the fact that first few eigenvectors of symbolic PCA account for highest variance of training samples and these few eigenvectors are enough to represent image for recognition purposes. Hence, improved recognition results can be achieved at less computational cost by using symbolic PCA, by virtue of its low dimensionality.

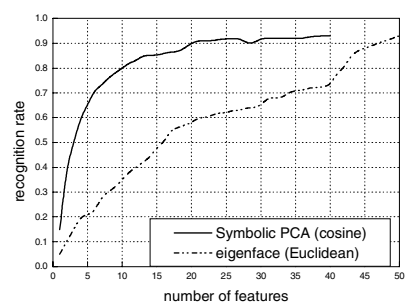

Fig. 3. Comparison of symbolic PCA and eigenface method for varying number of features

\section{Conclusion}

In this paper, the symbolic PCA approach for face recognition is presented and compared with eigenface method. The experimentation is done using ORL face database. The recognition accuracy obtained by symbolic PCA is better than that obtained by eigenface method for face images with variations in orientations, facial expressions and illuminations. By virtue of dimensionality reduction achieved in symbolic PCA, the improved recognition rates are attained at lower computation cost as compared to eigenface method. Hence, the proposed symbolic PCA is efficient and robust, and can be effectively used in face recognition systems. Further, the usage of symbolic similarity measures in the analysis will be considered in our future studies, in order to exploit the full strength of the symbolic data analysis approach. 


\section{Acknowledgement}

The authors are grateful to the referees for their valuable comments and suggestions. Also, the authors are indebted to Prof.K.Chidananda Gowda, Vice-chancellor, Kuvempu University, Karnataka, for his helpful discussions and encouragement. Further, the authors are also grateful to Dr.P.Nagabhushan, Professor, and Dr.D.S.Guru, Dept. of Computer Science, University of Mysore, Mysore, for their useful suggestions and encouragement.

\section{References}

1. Bock, H.H., Diday, E. (Eds.): Analysis of Symbolic Data. Springer Verlag (2000).

2. Choukria, Diday, Cazes: Extension of the principal component analysis to interval data. NTTS'95: New Techniques and Technologies for statistics, Bonn (1995).

3. Ichino, Yaguchi: Generalized Minkowski metrics for mixed feature type data analysis, vol(4).IEEE Trans. Systems Man Cybernet. (1994) 698-708.

4. Jain, Dubes: Algorithms for clustering data. Prentice-Hall, Englewood Cliffs, NJ (1998).

5. Kirby, Sirovich: Applications of the Karhunen-Loeve procedure for the characterization of human faces, v-12(1). IEEE Trans. Pattern Anal. Machine Intell. (1990)103-108.

6. Nagabhushan, Gowda, Diday: Dimensionality reduction of symbolic data, vol -16. Pattern Recognition Letters, (1995) 219-223.

7. Sudhanva, Gowda: Dimensionality reduction using geometric projection: a new technique, vol-5(8). Pattern Recognition (1995).

8. Turk, Pentland: Eigenfaces for Recognation, J Cognitive Neuro Science, (1991) 71-86 